\title{
BMJ Open Role of maintenance strategies in advanced epithelial ovarian cancer: a systematic review, network meta- analysis and cost-effectiveness analysis protocol
}

\author{
Qiancheng Hu, ${ }^{1}$ Wenli Kang, ${ }^{2}$ Qiuji Wu, ${ }^{1}$ Xin Wang, ${ }^{1}$ Qingfeng Wang, ${ }^{1}$ Ting Luo (1) ${ }^{3}$
}

To cite: Hu Q, Kang W, Wu Q, et al. Role of maintenance strategies in advanced epithelial ovarian cancer: a systematic review, network meta-analysis and cost-effectiveness analysis protocol. BMJ Open 2021;11:e051037. doi:10.1136/ bmjopen-2021-051037

- Prepublication history and additional supplemental material for this paper are available online. To view these files, please visit the journal online (http://dx.doi.org/10.1136/ bmjopen-2021-051037).

QH and WK contributed equally.

Received 10 March 2021 Accepted 23 November 2021

A) Check for updates

C Author(s) (or their employer(s)) 2021. Re-use permitted under CC BY-NC. No commercial re-use. See rights and permissions. Published by BMJ.

1Department of Abdominal Oncology, Cancer Center, West China Hospital, Sichuan University, Chengdu, China ${ }^{2}$ International Medical Center, West China Hospital, Sichuan University, Chengdu, China ${ }^{3}$ Department of Head, Neck and Mammary Gland Oncology, Cancer Center, West China Hospital, Sichuan University, Chengdu, China

Correspondence to

DrTing Luo; tina621@163.com

\section{ABSTRACT}

Introduction Different maintenance strategies have shown efficacy in patients with advanced ovarian cancer, but without drawing any conclusion on which strategy is preferred. Therefore, we will conduct a network metaanalysis and cost-effectiveness analysis to investigate maintenance strategies containing chemotherapy, poly (ADP-ribose) polymerase (PARP) inhibitors and antiangiogenesis therapy for patients with advanced ovarian cancer.

Methods and analysis The search strategy to identify potentially relevant studies will include hand searches in EMBASE, PubMed, Cochrane library and Web of science. The primary outcome is progression-free survival, defined as the date of randomisation to the date of progression or death. The secondary outcome is overall survival (calculated from the time from randomisation to death from any cause), grade 3-4 haematological and nonhaematological toxicities, quality-adjusted life years and incremental cost-effectiveness ratios. Two steps of meta-analysis will be carried out, traditional pair-wise meta-analysis and network meta-analysis. Methodological quality, risk of bias and the strength of evidence from randomised controlled trials (RCTs) will be proposed to assess the quality of RCTs. Heterogeneity, publication bias, subgroup analysis and sensitivity analysis will be explored. Ethics and dissemination The purpose of our study is to perform a comprehensive efficacy, safety and costeffectiveness analysis of all maintenance strategies in patients with advanced ovarian cancer. The results will be disseminated through international conference reports and peer-reviewed manuscripts. Ethics approval is not required for network meta-analysis and cost-effectiveness analysis. PROSPERO registration number CRD42021231814.

\section{INTRODUCTION}

Ovarian cancer (OC) is the seventh most common cancer in women and the fifth most lethal of all cancers among US women, accounting for nearly $5 \%$ of all cancers. ${ }^{1-3}$ $60 \%-70 \%$ of patients present with advanced disease $^{45}$ and the 5-year survival rate for Federation Internationale of Gynecologie
Strengths and limitations of this study

- In order to compare rankings of maintenance strategies in patients with advanced ovarian cancer, network meta-analysis will be performed in comparing the efficacy, safety and cost outcomes.

- A model-based cost-effectiveness analysis will be conducted using the indirect method based on a common comparator in our network meta-analysis.

- Methodological quality, risk of bias and the strength of evidence from randomised controlled trials (RCTs) will be proposed to assess the quality of RCTs by two reviewers independently.

- We will carry out the following subgroup analyses when possible: newly diagnosed and recurrent, platinum-sensitive and platinum-resistant, wildtype and mutant-type BRCA, homologous repair deficiency positive and negative, and Federation Internationale of Gynecologie and Obstetrigue (FIGO) II-III and FIGO IV.

- One possible limitation of this study is that only RCTs in English will be included in our network meta-analysis.

and Obstetrigue (FIGO) stage III and stage IV disease is $15 \% .^{5}$ Maximal cytoreductive surgery followed by platinum-based chemotherapy is the current standard of care in patients with advanced OC. ${ }^{6}$ Patients with advanced OC treated with platinum/paclitaxel as first-line chemotherapy often respond well, ${ }^{7}$ but the subsequent chemotherapy-free interval before disease progression is usually less than 12 months. ${ }^{8-10}$ Maintenance therapy with chemotherapy, poly (ADP-ribose) polymerase (PARP) inhibitor and antiangiogenesis agents is effective in prolonging progression-free interval. ${ }^{11-14}$

Vascular endothelial growth factor (VEGF) plays an important angiogenic role in advanced OC. By binding to VEGF-1/2 receptor, VEGF activates a downstream signal 
transduction cascade by intracellular tyrosine kinases. ${ }^{15}$ Anti-VEGF monoclonal antibodies and small molecule tyrosine kinase inhibitors have been developed to target cell signalling pathways involved in angiogenesis. ${ }^{16}$ Homologous repair deficiency (HRD) repair is another different mechanism of action, with protecting DNA double strands breaks. ${ }^{17}$ A PARP inhibitor inhibits catalytic domain and avoids the link between PARP and single strand of DNA. ${ }^{17}$ Researchers have increased the interest in combining PARP inhibitor and anti-angiogenesis agent treatment, because PARP inhibitors increase suppression of angiogenesis, leading to hypoxia and simultaneously hypoxia enhances PARP inhibitors. ${ }^{18}$

Several pairwise meta-analyses and network metaanalyses have examined the efficacy and safety of chemotherapy, ${ }^{19}$ anti-angiogenesis therapy ${ }^{20}$ and PARP inhibitors $^{21-23}$ as maintenance therapy either separately or collectively ${ }^{24-28}$ for patients with advanced OC. However, the following clinical characteristics of patients with advanced OC in pairwise and network meta-analyses are quite different, such as newly diagnosed and recurrent, platinum-sensitive and platinum-resistant, wild-type and mutant-type BRCA, HRD positive and negative, and FIGO II-III and FIGO IV. On the other hand, a recent pairwise meta-analysis showed that the same advantage, long-term survival rate (10 years), was not observed in patients with BRCA-mutated advanced $\mathrm{OC},{ }^{29}$ despite the improved short-term survival rate (5 years) regardless of BRCA mutational status in several previous pairwise meta-analyses. ${ }^{22}{ }^{26}$ Furthermore, the previous pairwise and network meta-analyses have only partly compared effectiveness and safety for patients with advanced OC, but they have not incorporated cost-effectiveness analysis and recent alternative treatments. ${ }^{30}$ The previous studies showed that PARP inhibitor and bevacizumab maintenance therapy were not cost effective for patients with advanced OC. ${ }^{31-33}$ The mean cost per patient for the PARP inhibitor for all strategy was 1.4-1.7 times higher than biomarker-directed PARP inhibitor front-line maintenance strategy. ${ }^{34}$

We will conduct a network meta-analysis and costeffectiveness analysis to investigate maintenance strategies containing chemotherapy, PARP inhibitors and anti-angiogenesis therapy for patients with advanced OC, and calculate a ranking value based on efficacy, safety and cost.

\section{METHODS}

The study will be developed following Preferred Reporting Items for Systematic Review and Meta-Analysis Protocols (PRISMA-P) ${ }^{35} 36$ and the Consolidated Health Economic Evaluation Reporting Standards. ${ }^{37}$ This protocol has been registered in PROSPERO. The study period is 2 years after registration of the protocol (registration date: 18 January 2021-18 February 2021; full study duration: January 2021-December 2022).

\section{Elgibility criteria}

Randomised controlled trials (RCTs) meeting the Population Intervention Comparison Outcomes Study Design statement described below will be included in this network meta-analysis.

\section{Participants (P)}

Women, over 18 years old, diagnosed with FIGO stage II-IV epithelial ovarian, fallopian tube and peritoneum carcinoma will be included in the study, regardless of newly diagnosed and recurrent, platinum-sensitive and platinum-resistant, wild-type and mutant-type BRCA, and HRD positive and negative.

\section{Interventions/comparators (I/C)}

All available maintenance strategies containing chemotherapy, PARP inhibitors and anti-angiogenesis will be considered as interventions. According to our previous search strategies in the literature, the interventions may be (1) paclitaxel, (2) 5-fluorouracil and cisplatin, (3) topotecan, (4) doxorubicin, (5) epidoxorubicin, (6) docetaxel, (7) nintedanib, (8) cediranib, (9) pazopanib, (10) bevacizumab, (11) trebananib, (12) sorafenib, (13) olaparib, (14) niraparib, (15) rucaparib, (16) erlotinib, (17) tanomastat, (18) abagovomab, (19) oregovomab, (20) lonafarnib and (21) placebo.

\section{Outcomes (0)}

The primary outcome is progression-free survival (PFS), defined as the date of randomisation to the date of progression or death. The secondary outcome is overall survival (calculated from the time from randomisation to death from any cause), grade 3-4 haematological and non-haematological toxicities, quality-adjusted life years (QALYs) and incremental cost-effectiveness ratios (ICERs). Grade 3-4 haematological and nonhaematological toxicities are performed in line with the Common Terminology Criteria for Adverse Events. ${ }^{38} 39$ Studies should provide at least one of the above outcomes.

\section{Study design (S)}

Phase II and III RCTs compare any two or more different interventions as alone or in combination in patients with advanced epithelial OC. There are no limitations in nationality distribution. The studies will be limited to results published in English language.

\section{Data sources and search strategy}

The search strategy to identify potentially relevant studies will include hand searches in EMBASE, PubMed, Cochrane library and Web of science. Electronic searching will be performed in major conferences proceedings according to titles of maintenance therapy for advanced OC, such as the annual meetings of American Society of Clinical Oncology and European Society of Medical Oncology (2018-2020). Although preliminary searches have already been completed up to 31 December 2020, the search will be updated regularly to include additional trials. Two reviewers who have been trained on the 
review process will independently assess all the potentially eligible studies based on inclusion criteria. The same two authors will screen the titles of reference from relevant reviews and meta-analysis to identify additional potential papers. If duplicate publications are identified, the latest data will be included. Any discrepancies will be resolved by discussion, or by consulting a third author. The results of the study selection and the reasons for excluding the full text will be presented using a PRISMA-compliant flow diagram. ${ }^{40}$ Full details of the search strategies in PubMed are outlined in more detail in online supplemental appendix 1.

We will use EndNote V.X7 to store and manage all literature searching records. A standardised form will be created to extract the following data in Microsoft Excel 2010 (Microsoft Corp, Redmond, Washington, USA, www.microsoft.com): the first author, study name, study design, publication time, total sample size, population, treatment arm, control arm, primary endpoint, follow-up duration and outcomes (HR for overall survival (OS) and progression-free survival (PFS), cost and grade 3-4 haematological and non-haematological toxicities). We will contact corresponding authors and relevant pharmaceutical companies for further information if important data are not reported in articles.

\section{Risk of bias}

The quality of individual RCTs will be assessed independently by two reviewers according to the following domains as outlined in the Cochrane Handbook V.5.1.0. ${ }^{41}$ The Handbook uses seven items (random sequence generation, allocation concealment, blinding of participants and personnel, incomplete outcome data, selective outcome reporting and other sources of bias) to rate the methodological quality as high risk, unclear risk and low risk for each outcome. All disagreement will be resolved through discussion or involving a third review author.

\section{Quality of evidence}

Two reviewers will independently use the Grades of Recommendation, Assessment, Development and Evaluation (GRADE) handbook to assess the quality of the body of evidence. ${ }^{42}$ The Handbook includes risk of bias, imprecision, inconsistency, indirectness and publication bias. The overall quality is classified as high, moderate, low and very low levels. For RCTs, the starting confidence level for each item is high but will be rated down based on the evaluation of the five domains. We will complete the GRADE process with GRADEprofiler software (V.3.6.1) ( www.gradeworkinggroup.org).

\section{Assessment of publication bias}

Comparison-adjusted funnel plot methods are constructed by Begg's and Egger's tests to help distinguish asymmetry, if available, due to test small study effects and publication bias. $^{43}$

\section{Statistical analysis}

We will perform traditional pair-wise meta-analysis for each arm with at least two studies, using Stata V.13.0 (StataCorp, College Station, Texas, USA). A network plot will be constructed consisting of vertices and edges between these nodes which are weighted by number of studies for the corresponding comparison. In addition, a contribution plot will also be constructed to present the per cent contribution of direct and indirect comparisons to the entire network meta-analytic summary effects. For each type of maintenance strategies in patients with advanced OC, network meta-analysis will be mainly performed using OpenBUGS (V.3.2.3). Results regarding grade 3-4 haematological and non-haematological toxicities are expressed as ORs for dichotomous outcomes with $95 \%$ CIs/credible intervals. Pooled HRs for OS and PFS with 95\% CIs will be calculated. A random-effects model will be run in each outcome of interest, and the stability of the results will be recalculated using a fixedeffects model to explore further where there is evidence of heterogeneity. ${ }^{44}$ We will select the final model as the best-fit model to pool the data with the lowest deviance information criterion. ${ }^{45}$ 46 $\mathrm{A}$ consistency model will be adopted for network meta-analysis with no obvious inconsistency; otherwise, an inconsistency model will be employed. ${ }^{47}$ The node-splitting analysis and loop-specific approach will be used to assess inconsistency between direct and indirect treatment effects. ${ }^{48} 49$ The group with the surface under the cumulative ranking (SUCRA) of being the most effective in term of OS, PFS, safety, QALYs and ICERs will be assessed in the network meta-analysis, with higher SUCRA indicating better efficacy and safety. The multidimensional cluster analysis will be conducted to assess comprehensive efficacy, tolerability and cost effectiveness of maintenance strategies based on SUCRA value. Heterogeneity will be assessed with the $\mathrm{I}^{2}$ statistic, where $\mathrm{I}^{2}$ less than $25 \%$ indicated low heterogeneity. ${ }^{50} \mathrm{~A}$ two-sided $\mathrm{p}<0.05$ is considered statistically significant.

\section{Cost-effectiveness analysis}

We will assess the most cost-effective maintenance therapy regimens in patients with advanced OC from the perspective of the third-party payer in the USA. All modelling and calculations will be implemented using TreeAge Pro 2009 software (Williamstown, Massachusetts, USA). The main outputs of the model are life-years, QALYs and ICER. The structure of the Markov model comprises three mutually exclusive health states: PFS (with or without maintenance treatment), progression and death. The expected total costs, QALYs and ICERs will be estimated over a lifetime horizon (10 years). Plot Digitizer software (V.2.6.8) will be used to extract available data points from the PFS and OS Kaplan-Meier curves, if they are presented as figures rather than numbers. All health utility values of PFS and progressed disease (PD) health status are obtained from previously published cost-effectiveness studies. ${ }^{32}{ }^{33} \mathrm{All}$ relevant costs will be taken into account, such as direct medical costs (hospitalisation costs, pharmacy costs and 
medical treatment costs), direct non-medical costs (transportation costs) and indirect costs (costs of production loss). The pharmacy costs of maintenance therapy will be obtained from the wholesale prices paid by the pharmacy of West China Hospital. All costs will be adjusted to represent 2020 US dollars using Tom's Inflation Calculator (https://www.halfhill.com/inflation_js.html, 2020). Costs and outcomes will be discounted at $3 \%$ annually. ${ }^{51}$ A willingness to pay (WTP) of less than three times country's gross domestic product per capita of China in 2020 (\$31 512) will be used as a threshold to determine cost effectiveness. One-way sensitivity analysis and probabilistic sensitivity analyses will be performed to assess the potential drivers of economic outcomes. Subgroup analysis will also be performed in patients categorised by BRCA and HRD status.

\section{Transitivity, homogeneity and consistency assumption}

To achieve valid results, we will perform three key assumptions underlying the network meta-analysis (transitivity, homogeneity and consistency assumption). ${ }^{52}$ First, we will conduct a thorough comparison of important study and patient characteristics. Second, we will perform a multivariate meta-regression analysis to examine possible sources of heterogeneity using the same interventions. Lastly, indirect evidence via a common comparator is not different from direct evidence in the network. ${ }^{49} 53$

\section{Subgroup analysis}

We categorise maintenance therapy regimens into the following groups when possible: newly diagnosed and recurrent, platinum-sensitive and platinum-resistant, wild-type and mutant-type BRCA, HRD positive and negative, and FIGO II-III and FIGO IV.

\section{Sensitivity analysis}

In order to assess the robustness and reliability of findings in our studies, the following sensitivity analyses will be conducted for the outcomes of interest (if appropriate). The first sensitivity analysis will be performed excluding maintenance therapy regimens that have not been approved by any food and drug administration. The second sensitivity analysis will restrict random-effects and fixed-effects models and study design (phase II vs phase III RCTs). Furthermore, if RCTs have a high risk of bias, it will be excluded from the analysis. Lastly, we will perform the sensitivity analysis by excluding one paper at a time and explore the heterogeneity of the findings. ${ }^{54}$

\section{Patient and public involvement}

Patients and the public will be not involved in the design, conduct, reporting or dissemination of this study.

\section{Ethics and dissemination}

This study does not involve human participants. There is no need to obtain formal ethical approval, given that the network meta-analysis comes from openly available databases. The results will be disseminated through international conference reports and peer-reviewed manuscripts.

\section{DISCUSSION}

Despite recent improvements in the maintenance strategies of advanced OC, in the worldwide, less than $40 \%$ of patients with OC can be cured. ${ }^{55}$ Thus, identifying the most effective maintenance strategies remains a challenge for researchers, patients and policy-makers when making decisions between multiple possible alternative maintenance strategies to prevent advanced OC. Compared with the results from existing multiple separate pairwise meta-analyses, network meta-analysis and cost-effectiveness analysis, these findings will be crucial to inform evidence-based priorities and enhance decision-making in patients with advanced OC.

The role of maintenance strategies is a rapidly evolving field, and PARP inhibitor and anti-angiogenesis agents have already shown that PFS was significantly increased when compared with chemotherapy alone. ${ }^{121356}$ Recently, olaparib plus bevacizumab as maintenance therapy has shown a longer PFS (22.1 m vs $16.6 \mathrm{~m}, 95 \%$ CI 0.49 to $0.72, \mathrm{p}<0.001$ ) compared with olaparib alone in patient with newly diagnosed, advanced, high-grade OC. ${ }^{30}$ Yet, as a type of targeted therapy, there are still important questions, including the selection of patients based on BRCA1/2 mutations, HRD positive or platinum sensitivity (or not), the duration of maintenance treatment and whether maintenance therapy should be used as monotherapy or in combination.

The biomarker-directed use of maintenance therapy might improve PFS but not be associated with high healthcare costs in patients with advanced OC. ${ }^{34}$ Treatment with maintenance bevacizumab was not cost effective, because it was associated with improved PFS but led to slightly higher healthcare costs in patients with advanced OC. ${ }^{33} \mathrm{~A}$ cost-effectiveness analysis performed by Gonzalez et al demonstrated that adopting a biomarker-directed maintenance strategy was cost effective compared with a PARP inhibitor-for-all approach in patients with newly diagnosed advanced OC. ${ }^{34}$

The purpose of our study is to perform a comprehensive efficacy, safety and cost-effectiveness analysis of all maintenance strategies in patients with advanced OC. It is hoped to explore target subset populations to enhance the efficiency and quality of maintenance therapy. Maintenance therapy is warranted to not only target patients who will most likely benefit from treatment but also decrease unnecessary toxicities that occur from using drugs while decreasing the cost of cancer care in patients with advanced OC.

Contributors $\mathrm{QH}$ and QWu conceived the presented idea. QH and WK participated in the development of the search strategy for this study. $\mathrm{QH}$ wrote and reviewed the manuscript. XW and QWa contributed to the study design and critical revision of the manuscript. TL was serving as a guarantor for the content and corresponding author of this article. All authors read and approved the final manuscript.

Funding This work was supported by the project of Sichuan Department of Science and Technology (grant number: 2021YES0235).

Competing interests None declared.

Patient consent for publication Not applicable.

Provenance and peer review Not commissioned; externally peer reviewed.

Supplemental material This content has been supplied by the author(s). It has not been vetted by BMJ Publishing Group Limited (BMJ) and may not have been peer-reviewed. Any opinions or recommendations discussed are solely those of the author(s) and are not endorsed by BMJ. BMJ disclaims all liability and 
responsibility arising from any reliance placed on the content. Where the content includes any translated material, BMJ does not warrant the accuracy and reliability of the translations (including but not limited to local regulations, clinical guidelines, terminology, drug names and drug dosages), and is not responsible for any error and/or omissions arising from translation and adaptation or otherwise.

Open access This is an open access article distributed in accordance with the Creative Commons Attribution Non Commercial (CC BY-NC 4.0) license, which permits others to distribute, remix, adapt, build upon this work non-commercially, and license their derivative works on different terms, provided the original work is properly cited, appropriate credit is given, any changes made indicated, and the use is non-commercial. See: http://creativecommons.org/licenses/by-nc/4.0/.

\section{ORCID iD}

Ting Luo http://orcid.org/0000-0002-2923-9344

\section{REFERENCES}

1 Webb PM, Jordan SJ. Epidemiology of epithelial ovarian cancer. Best Pract Res Clin Obstet Gynaecol 2017;41:3-14.

2 Chen W, Zheng R, Baade PD, et al. Cancer statistics in China, 2015. CA Cancer J Clin 2016;66:115-32.

3 Siegel RL, Miller KD, Jemal A. Cancer statistics, 2019. CA Cancer J Clin 2019;69:7-34.

4 Smith RA, Andrews KS, Brooks D, et al. Cancer screening in the United States, 2018: a review of current American cancer Society guidelines and current issues in cancer screening. CA Cancer J Clin 2018;68:297-316.

5 Jessmon P, Boulanger T, Zhou W, et al. Epidemiology and treatment patterns of epithelial ovarian cancer. Expert Rev Anticancer Ther 2017;17:427-37.

6 Wright AA, Bohlke K, Armstrong DK, et al. Neoadjuvant chemotherapy for newly diagnosed, advanced ovarian cancer: Society of gynecologic oncology and American Society of clinical oncology clinical practice guideline. Gynecol Oncol 2016;143:3-15.

7 Jayson GC, Kohn EC, Kitchener HC, et al. Ovarian cancer. Lancet 2014;384:1376-88

8 Hanker LC, Loibl S, Burchardi N, et al. The impact of second to sixth line therapy on survival of relapsed ovarian cancer after primary taxane/platinum-based therapy. Ann Oncol 2012;23:2605-12.

9 Ledermann J, Harter P, Gourley C, et al. Olaparib maintenance therapy in patients with platinum-sensitive relapsed serous ovarian cancer: a preplanned retrospective analysis of outcomes by BRCA status in a randomised phase 2 trial. Lancet Oncol 2014;15:852-61.

10 Ledermann JA, Hackshaw A, Kaye S, et al. Randomized phase II placebo-controlled trial of maintenance therapy using the oral triple angiokinase inhibitor BIBF 1120 after chemotherapy for relapsed ovarian cancer. J Clin Oncol 2011;29:3798-804.

11 Pecorelli S, Favalli G, Gadducci A, et al. Phase III trial of observation versus six courses of paclitaxel in patients with advanced epithelial ovarian cancer in complete response after six courses of paclitaxel/ platinum-based chemotherapy: final results of the After- 6 protocol 1. $J$ Clin Oncol 2009;27:4642-8.

12 Pujade-Lauraine E, Ledermann JA, Selle F, et al. Olaparib tablets as maintenance therapy in patients with platinum-sensitive, relapsed ovarian cancer and a BRCA1/2 mutation (SOLO2/ENGOT-Ov21): a double-blind, randomised, placebo-controlled, phase 3 trial. Lancet Oncol 2017; 18:1274-84

13 Burger RA, Brady MF, Bookman MA, et al. Incorporation of bevacizumab in the primary treatment of ovarian cancer. $N$ Engl $J$ Med 2011:365:2473-83.

14 Perren TJ, Swart AM, Pfisterer J, et al. A phase 3 trial of bevacizumab in ovarian cancer. N Engl J Med 2011;365:2484-96

15 García García Y, Marín Alcalá M, Martínez Vila C. Anti-angiogenic therapy for ovarian cancer. EJC Suppl 2020;15:77-86.

16 Choi H-J, Armaiz Pena GN, Pradeep S, et al. Anti-vascular therapies in ovarian cancer: moving beyond anti-VEGF approaches. Cancer Metastasis Rev 2015;34:19-40.

17 Evans T, Matulonis U. PARP inhibitors in ovarian cancer: evidence, experience and clinical potential. Ther Adv Med Oncol 2017;9:253-67

18 Liu JF, Barry WT, Birrer M, et al. Combination cediranib and olaparib versus olaparib alone for women with recurrent platinum-sensitive ovarian cancer: a randomised phase 2 study. Lancet Oncol 2014:15:1207-14

19 Hess LM, Rong N, Monahan PO, et al. Continued chemotherapy after complete response to primary therapy among women with advanced ovarian cancer: a meta-analysis. Cancer 2010;116:5251-60.
20 Jiang $\mathrm{Y}$, Sun $\mathrm{X}$, Kong B, et al. Antiangiogenesis therapy in ovarian cancer patients: an updated meta-analysis for 15 randomized controlled trials. Medicine 2018;97:e11920.

21 Staropoli N, Ciliberto D, Del Giudice T, et al. The Era of PARP inhibitors in ovarian cancer: "Class Action" or not? A systematic review and meta-analysis. Crit Rev Oncol Hematol 2018;131:83-9.

22 Tomao F, Bardhi E, Di Pinto A, et al. Parp inhibitors as maintenance treatment in platinum sensitive recurrent ovarian cancer: an updated meta-analysis of randomized clinical trials according to BRCA mutational status. Cancer Treat Rev 2019;80:101909.

23 Ibrahim EM, Refae AA, Bayer AM, et al. Poly(ADP-ribose) polymerase inhibitors as maintenance treatment in patients with newly diagnosed advanced ovarian cancer: a meta-analysis. Future Oncol 2020;16:585-96.

24 Qian X, Qin J, Pan S, et al. Maintenance therapy in ovarian cancer with targeted agents improves pfs and OS: a systematic review and meta-analysis. PLoS One 2015;10:e0139026.

$25 \mathrm{Xu}$ X, Yin S, Guo H, et al. Comparative efficacy of targeted maintenance therapy for newly diagnosed epithelial ovarian cancer: a network meta-analysis. Cancer Manag Res 2019;11:4119-28.

26 Stemmer A, Shafran I, Stemmer SM, et al. Comparison of poly (ADPribose) polymerase inhibitors (PARPis) as maintenance therapy for platinum-sensitive ovarian cancer: systematic review and network meta-analysis. Cancers 2020;12. doi:10.3390/cancers 12103026 . [Epub ahead of print: 1810 2020].

27 Bartoletti M, Pelizzari G, Gerratana L, et al. Bevacizumab or PARPInhibitors maintenance therapy for platinum-sensitive recurrent ovarian cancer: a network meta-analysis. Int J Mol Sci 2020;21:3805

28 Feng $\mathrm{Y}$, Huang $\mathrm{H}$, Wan $\mathrm{T}$, et al. Comparison of PARPis with angiogenesis inhibitors and chemotherapy for maintenance in ovarian cancer: a network meta-analysis. Adv Ther 2019;36:3368-80.

29 Nahshon C, Barnett-Griness O, Segev Y, et al. Five-year survival decreases over time in patients with BRCA-mutated ovarian cancer: a systemic review and meta-analysis. Int J Gynecol Cancer 2020. doi:10.1136/ijgc-2020-001392. [Epub ahead of print: 09 Jun 2020].

30 Ray-Coquard I, Pautier P, Pignata S, et al. Olaparib plus bevacizumab as first-line maintenance in ovarian cancer. $N$ Engl $J$ Med 2019;381:2416-28.

31 Smith HJ, Walters Haygood CL, Arend RC, et al. PARP inhibitor maintenance therapy for patients with platinum-sensitive recurrent ovarian cancer: a cost-effectiveness analysis. Gynecol Oncol 2015;139:59-62.

32 Dottino JA, Moss HA, Lu KH, et al. U.S. food and drug Administration-approved poly (ADP-ribose) polymerase inhibitor maintenance therapy for recurrent ovarian cancer: a costeffectiveness analysis. Obstet Gynecol 2019;133:795-802.

33 Cohn DE, Kim KH, Resnick KE, et al. At what cost does a potential survival advantage of bevacizumab make sense for the primary treatment of ovarian cancer? A cost-effectiveness analysis. J Clin Oncol 2011;29:1247-51.

34 Gonzalez R, Havrilesky LJ, Myers ER, et al. Cost-effectiveness analysis comparing "PARP inhibitors-for-all" to the biomarkerdirected use of PARP inhibitor maintenance therapy for newly diagnosed advanced stage ovarian cancer. Gynecol Oncol 2020;159:483-90.

35 Moher D, Liberati A, Tetzlaff J, et al. Preferred reporting items for systematic reviews and meta-analyses: the PRISMA statement. PLoS Med 2009;6:e1000097.

36 Moher D, Shamseer L, Clarke M, et al. Preferred reporting items for systematic review and meta-analysis protocols (PRISMA-P) 2015 statement. Syst Rev 2015;4:1.

37 Husereau D, Drummond M, Petrou S, et al. Consolidated Health Economic Evaluation Reporting Standards (CHEERS)--explanation and elaboration: a report of the ISPOR Health Economic Evaluation Publication Guidelines Good Reporting Practices Task Force. Value Health 2013:16:231-50.

38 Trotti A, Colevas AD, Setser A, et al. Patient-reported outcomes and the evolution of adverse event reporting in oncology. $J$ Clin Oncol 2007:25:5121-7.

39 Thor M, Deasy JO, Paulus R, et al. Tolerance doses for late adverse events after hypofractionated radiotherapy for prostate cancer on trial NRG Oncology/RTOG 0415. Radiother Oncol 2019;135:19-24.

40 Moher D, Liberati A, Tetzlaff J, et al. Preferred reporting items for systematic reviews and meta-analyses: the PRISMA statement. BMJ 2009:339:b2535.

41 Higgins JPT, Altman DG, Gøtzsche PC, et al. The Cochrane collaboration's tool for assessing risk of bias in randomised trials. BMJ 2011;343:d5928.

42 Atkins D, Eccles M, Flottorp S, et al. Systems for grading the quality of evidence and the strength of recommendations I: critical appraisal 
of existing approaches the grade Working group. BMC Health Serv Res 2004;4:38.

43 Egger M, Davey Smith G, Schneider M, et al. Bias in meta-analysis detected by a simple, graphical test. BMJ 1997;315:629-34.

44 Cameron C, Coyle D, Richter T, et al. Systematic review and network meta-analysis comparing antithrombotic agents for the prevention of stroke and major bleeding in patients with atrial fibrillation. BMJ Open 2014;4:e004301.

45 Qiu Z, Song PXK, Tan M. Bayesian hierarchical models for multilevel repeated ordinal data using WinBUGS. J Biopharm Stat 2002;12:121-35.

46 Zintzaras E, loannidis JPA. Heterogeneity testing in meta-analysis of genome searches. Genet Epidemiol 2005;28:123-37.

47 Xu C, Chen Y-P, Du X-J, et al. Comparative safety of immune checkpoint inhibitors in cancer: systematic review and network metaanalysis. BMJ 2018;363:k4226.

48 Dias S, Welton NJ, Caldwell DM, et al. Checking consistency in mixed treatment comparison meta-analysis. Stat Med 2010;29:932-44.

49 Higgins JPT, Jackson D, Barrett JK, et al. Consistency and inconsistency in network meta-analysis: concepts and models for multi-arm studies. Res Synth Methods 2012;3:98-110.

50 Mao L, Jian C, Changzhi L, et al. Cytochrome CYP2C19 polymorphism and risk of adverse clinical events in clopidogrel- treated patients: a meta-analysis based on 23,035 subjects. Arch Cardiovasc Dis 2013;106:517-27.

51 Sanders GD, Neumann PJ, Basu A, et al. Recommendations for conduct, methodological practices, and reporting of costeffectiveness analyses: second panel on cost-effectiveness in health and medicine. JAMA 2016;316:1093-103.

52 Song F, Loke YK, Walsh T, et al. Methodological problems in the use of indirect comparisons for evaluating healthcare interventions: survey of published systematic reviews. BMJ 2009;338:b1147.

53 Tesfamariam Y, Jakob T, Wöckel A, et al. Adjuvant bisphosphonates or RANK-ligand inhibitors for patients with breast cancer and bone metastases: a systematic review and network meta-analysis. Crit Rev Oncol Hematol 2019;137:1-8.

54 Zacher J, Kasenda B, Engert A, et al. The role of additional radiotherapy for primary central nervous system lymphoma. Cochrane Database Syst Rev 2014;6:CD009211.

55 Siegel RL, Miller KD, Jemal A. Cancer statistics, 2018. CA Cancer J Clin 2018;68:7-30.

56 Burger RA, Brady MF, Rhee J, et al. Independent radiologic review of the gynecologic Oncology Group study 0218, a phase III trial of bevacizumab in the primary treatment of advanced epithelial ovarian, primary peritoneal, or fallopian tube cancer. Gynecol Oncol 2013;131:21-6. 